\title{
General Decay Anti-synchronization of Multi-weighted Coupled Neural Networks with and without Reaction-Diffusion Terms
}

\author{
Yanli Huang • Jie Hou · Erfu Yang
}

Received: date / Accepted: date

\begin{abstract}
The network models of multi-weighted coupled neural networks (MWCNNs) and multi-weighted coupled reaction-diffusion neural networks (MWCRDN$\mathrm{Ns}$ ) with and without delayed coupling are presented in this paper, respectively. Firstly, on account of the definitions of $\psi$-type stability and $\psi$-type function, the concept of decay anti-synchronization is proposed. Then, we investigate the decay anti-synchronization of MWCNNs with and without delayed coupling by designing appropriate nonlinear controllers, and several criteria for ensuring decay anti-synchronization are inferred by means of Lyapunov functional method as well as inequality techniques. Similarly, some conditions for decay anti-synchronization of MWCRDNNs with and without delayed coupling are also respectively derived. Lastly, two numerical examples with simulations are given to validate the correctness of these derived results.
\end{abstract}

Keywords General decay anti-synchronization . MWCNNs · Nonlinear control · Delayed coupling · Reaction-diffusion terms

Yanli Huang

School of Computer Science and Technology, Tianjin Key Laboratory of Optoelectronic Detection Technology and System, Tianjin Polytechnic University, Tianjin 300387, China E-mail: huangyanli@tjpu.edu.cn

Jie $\mathrm{Hou}$

School of Computer Science and Technology, Tianjin Polytechnic University, Tianjin 300387, China

Erfu Yang

Department of Design, Manufacture and Engineering Management, Faculty of Engineering, University of Strathclyde, Glasgow G1 1XJ, Scotland, UK

\section{Introduction}

In recent several decades, increasing attention has been paid to the study of neural networks $(\mathrm{NNs})$ in virtue of their potential applications in various fields, such as optimization, parameter estimation, pattern recognition and so on $[4,6,7,15,19,20,26,29,45,56,57]$. Accordingly, it has been a hot topic to discuss the dynamic behaviours (e.g., synchronization, passivity and stability) in NNs $[17,23,30,38,44,46,48]$. In [23], exponential $\mathcal{H}_{\infty}$ synchronization problem of NNs with discrete time delays was considered. The stability for a class of memristive NNs was concerned and several conditions were established for guaranteeing stability in [30]. Nevertheless, the aforementioned literatures neglect the phenomenon of reaction diffusion in NNs. Strictly speaking, the reaction-diffusion phenomenon in NNs is inevitable when electrons propagate in inhomogeneous electromagnetic fields. Therefore, it is significant to take reaction-diffusion terms into consideration in $\mathrm{NNs}$, and a number of pursuers have studied reaction-diffusion NNs (RDNNs) $[11,12,22,32]$. The authors discussed the passivity as well as stability of NNs with reaction-diffusion terms in [32].

In particular, when many NNs interconnect with each other, a special kind of complex networks, namely coupled NNs (CNNs), is taken shape. Recently, CNNs have been attracted wide attention because of their extensive applications in various prospects, such as brain science and secure communication. Consequently, a great quantity of meaningful research results on CNNs have been published $[10,27,28,42,49]$. In [10], the fixed-time synchronization for a kind of CNNs with parameter uncertainties was investigated. The authors discussed the synchronization of memristor-based recurrent CNNs with time-varying delays in [49]. In addition, there are some 
literatures on the study of coupled reaction-diffusion neural networks (CRDNNs) [31,33,39]. The synchronization and passivity of linearly CRDNNs with adaptive couplings were considered in [33]. In [31], the synchronization and $\mathcal{H}_{\infty}$ synchronization problems for an array of CRDNNs were considered.

It is well known that synchronization inhere in many practical systems, such as the generation and development of languages, an array of networks consisting of a set of identical delayed NNs and so on. Therefore, there are a large number of investigators devote themselves to studying the synchronization of CNNs and CRDNNs $[9,10,28,31,33,39,42,49]$. In fact, antisynchronization is also a fascinating phenomenon in the real world, which widely exists in memristive recurrent NNs, periodic oscillators, and so forth. Moreover, up to now, anti-synchronization has been successfully applied in many fields, for instance, image processing, information science and so on. Hence, it is highly meaningful to study anti-synchronization $[16,21,24,40,53]$. In [40], the anti-synchronization problem for a class of memristive CNNs was investigated. Nevertheless, only few papers have considered reaction-diffusion phenomena in the study of anti-synchronization [43]. The authors in [43] analyzed anti-synchronization of CRDNNs with time-varying delayed coupling.

As pointed out in [14], it is extremely useful and fascinating topic to estimate the solution's convergence rate of nonlinear systems. Actually, in many practical cases, the convergence time or speed of the system is hard to acquire. Consequently, the conception of decay synchronization is proposed, which is derived from decay stability ( $\psi$-type stability) $[1,25,35,36]$. In [25], the decay synchronization for a class of bidirectional associative memory NNs was investigated. The authors studied the decay synchronization of NNs with time delays in [1]. It is a pity that the decay antisynchronization of CNNs and CRDNNs has not been considered in existing research results.

It is worth noting that the network models in the aforementioned literatures are with single weight. In fact, a great many of existing networks are more accurately described by multi-weighted complex dynamical networks (MWCDNs), for instance, transportation networks, social networks, communication networks and so on. Hence, it is of great significance to study MWCDNs $[2,3,54,55]$. In [2], the global synchronization of MWCDNs was studied and a criterion was obtained for guaranteeing synchronization. The general decay synchronization of delayed MWCDNs was considered by constructing suitable nonlinear feedback controllers in [55]. As a particular sort of MWCDNs, multi-weighted CNNs (MWCNNs) have also been concerned in $[8,34]$. Wang et al. [34] considered finite-time passivity of MWCNNs and derived several finite-time synchronization conditions. Unfortunately, there is no research results reported on the decay anti-synchronization for MWCNNs as well as multi-weighted CRDNNs (MWCRDNNs) until now.

In view of the foregoing statement, we consider the general decay anti-synchronization of MWCNNs as well as MWCRDNNs, respectively. Firstly, the general decay anti-synchronization is defined by introducing $\psi$ type function. Additionally, we investigate the decay anti-synchronization for MWCNNs with and without delayed coupling by designing suitable nonlinear controllers and constructing appropriate Lyapunov functional, and some criteria for guaranteeing decay antisynchronization are obtained. Furthermore, we also discuss the decay anti-synchronization for MWCRDNNs with Dirichlet boundary conditions, and several decay anti-synchronization conditions for MWCRDNNs with and without delayed coupling are put forward.

\section{Preliminaries}

Definition 1 (see [41]) If the function $\psi(t): \mathbb{R}_{+} \rightarrow$ $(0,+\infty)$ satisfies the conditions as follows:

1) $\psi(t)$ is nondecreasing and differentiable;

2) $\psi(+\infty)=+\infty$ and $\psi(0)=1$;

3) $\widehat{\psi}(t):=\frac{\dot{\psi}(t)}{\psi(t)}$ is decreasing;

4) $\forall \alpha, \beta \geqslant 0, \psi(\alpha+\beta) \leqslant \psi(\alpha) \psi(\beta)$;

then it is called to be $\psi$-type function.

Lemma 1 (see [18]) Let $\Theta$ be a cube $\left|\vartheta_{r}\right|<\iota_{r}(r=$ $1,2, \cdots, p)$ and $Z(\vartheta) \in C^{1}(\Theta)$ be a real-valued function, which satisfies $\left.Z(\vartheta)\right|_{\partial \Theta}=0$. Then

$$
\int_{\Theta} Z^{2}(\vartheta) d \vartheta \leqslant \iota_{r}^{2} \int_{\Theta}\left(\frac{\partial Z}{\partial \vartheta_{r}}\right)^{2} d \vartheta
$$

where $\vartheta=\left(\vartheta_{1}, \vartheta_{2}, \cdots, \vartheta_{p}\right)^{T}$.

Notations: $\lambda(\cdot)$ signifies the eigenvalue of the corresponding matrix. For any $e(t)=\left(e_{1}(t), e_{2}(t), \cdots, e_{M}(t)\right)^{T}$ $\in \mathbb{R}^{M},\|e(t)\|=\sqrt{\sum_{s=1}^{M} e_{s}^{2}(t)}$. Moreover, if $e(\vartheta, t)=$ $\left(e_{1}(\vartheta, t), e_{2}(\vartheta, t), \cdots, e_{M}(\vartheta, t)\right)^{T} \in \mathbb{R}^{M}$, then $\|e(\cdot, t)\|_{2}=$ $\sqrt{\int_{\Theta} \sum_{s=1}^{M} e_{s}^{2}(\vartheta, t) d \vartheta}$, where $(\vartheta, t) \in \Theta \times \mathbb{R}$ and $\Theta=$ $\left\{\vartheta=\left(\vartheta_{1}, \vartheta_{2}, \cdots, \vartheta_{p}\right)^{T}|| \vartheta_{r} \mid<l_{r}, r=1,2, \cdots, p\right\} \subset$ $\mathbb{R}^{p}$. 


\section{General decay anti-synchronization of MWCNNs with and without delayed coupling}

3.1 General decay anti-synchronization of MWCNNs

Consider the following MWCNNs model:

$$
\begin{aligned}
\dot{Y}_{s}(t)= & -A Y_{s}(t)+B g\left(Y_{s}(t)\right)+c_{1} \sum_{j=1}^{M} G_{s j}^{1} \Gamma_{1} Y_{j}(t) \\
& +c_{2} \sum_{j=1}^{M} G_{s j}^{2} \Gamma_{2} Y_{j}(t)+\cdots \\
& +c_{m} \sum_{j=1}^{M} G_{s j}^{m} \Gamma_{m} Y_{j}(t), \quad s=1,2, \cdots, M,
\end{aligned}
$$

where $\mathbb{R} \ni M>0$ represents the total number of nodes in the network, $Y_{s}(t)=\left(Y_{s 1}(t), Y_{s 2}(t), \cdots, Y_{s n}(t)\right)^{T} \in$ $\mathbb{R}^{n}$ is the state vector of the $s$ th node; $A=\operatorname{diag}\left(a_{1}, a_{2}, \cdots\right.$ ,$\left.a_{n}\right) \in \mathbb{R}^{n \times n}>0, B \in \mathbb{R}^{n \times n}$ symbols a constant ma$\operatorname{trix} ; g\left(Y_{s}(t)\right)=\left(g_{1}\left(Y_{s 1}(t)\right), g_{2}\left(Y_{s 2}(t)\right), \cdots, g_{n}\left(Y_{s n}(t)\right)\right)^{T}$ $\in \mathbb{R}^{n}$ and $\mathbb{R} \ni c_{\kappa}>0(\kappa=1,2, \cdots, m)$ symbols coupling strength for the $\kappa$ th coupling form; $\Gamma_{\kappa} \in \mathbb{R}^{n \times n}(\kappa=$ $1,2, \cdots, m)$ is positive definite matrix, which symbols the inner coupling matrix of the $\kappa$ th coupling form; $G^{\kappa}=\left(G_{s j}^{\kappa}\right)_{M \times M} \in \mathbb{R}^{M \times M}(\kappa=1,2, \cdots, m)$ expresses coupling weight between nodes in the $\kappa$ th coupling form, where $G_{s j}^{\kappa}$ is defined as follows: $G_{s j}^{\kappa}=G_{j s}^{\kappa}>$ 0 if and only if there exists a connection between node $s$ and node $j$ for the $\kappa$ th coupling form; if not, $G_{s j}^{\kappa}=$ $G_{j s}^{\kappa}=0(s \neq j) ;$ and

$$
G_{s s}^{\kappa}=-\sum_{\substack{j=1 \\ j \neq s}}^{M} G_{s j}^{\kappa}, s=1,2, \cdots, M .
$$

Remark 1 It is well known that a large scale of networks in our real world should be represented by MWCDNs, in which nodes are coupled in the form of multiple coupling, such as social networks, communication networks, transportation networks and so on. Hence, it is of great significance to study MWCDNs $[2,3,54,55]$. An et al. [3] handled the problem of public traffic network from a new visual field of CDNs with multi-weights and analyzed the impact of congestion degrees, transfers coefficient and passenger flow density between different bus lines on the complex public traffic network to its synchronous ability. Moreover, MWCNNs is a special class of MWCDNs, which has also been studied recently $[8,34]$. The authors in [8] studied anti-synchronization and pinning control problem of MWCNNs. Unfortunately, there is no research results reported on the decay anti-synchronization for MWCNNs until now, which motivates our research work in this paper.
Consider the network model (1) as the drive system. Then its corresponding response system is introduced as:

$$
\begin{aligned}
\dot{W}_{s}(t)= & -A W_{s}(t)+B g\left(W_{s}(t)\right)+c_{1} \sum_{j=1}^{M} G_{s j}^{1} \Gamma_{1} W_{j}(t) \\
& +c_{2} \sum_{j=1}^{M} G_{s j}^{2} \Gamma_{2} W_{j}(t)+\cdots+c_{m} \sum_{j=1}^{M} G_{s j}^{m} \Gamma_{m} W_{j}(t) \\
& +u_{s}(t), \quad s=1,2, \cdots, N
\end{aligned}
$$

where $W_{s}(t)=\left(W_{s 1}(t), W_{s 2}(t), \cdots, W_{s n}(t)\right)^{T} \in \mathbb{R}^{n}$ is the state vector of the $s$ th node; $u_{s}(t)=\left(u_{s 1}(t), u_{s 2}(t), \cdots\right.$ , $\left.u_{s n}(t)\right)^{T} \in \mathbb{R}^{n}$ is the suitable controller to achieve a certain control objective; $A, B, c_{\kappa}, G_{s j}^{\kappa}, \Gamma_{\kappa}$ have the same definitions as in system (1).

Assumption 1 For any $\beta_{1}, \beta_{2} \in \mathbb{R}$, the function $g_{i}(\cdot)(i$ $=1,2, \cdots, n)$ satisfies

$$
\left|g_{i}\left(\beta_{1}\right)+g_{i}\left(\beta_{2}\right)\right| \leqslant F_{i}\left|\beta_{1}+\beta_{2}\right|,
$$

where $0<F_{i} \in \mathbb{R}$. Take $F=\operatorname{diag}\left(F_{1}^{2}, F_{2}^{2}, \cdots, F_{n}^{2}\right) \in$ $\mathbb{R}^{n \times n}$.

Take $e_{s}(t)=Y_{s}(t)+W_{s}(t)$. By (1) and (2), we can obtain

$$
\begin{aligned}
\dot{e}_{s}(t)= & -A e_{s}(t)+B g\left(Y_{s}(t)\right)+\sum_{\kappa=1}^{m} \sum_{j=1}^{M} c_{\kappa} G_{s j}^{\kappa} \Gamma_{\kappa} e_{j}(t) \\
& +B g\left(W_{s}(t)\right)+u_{s}(t) .
\end{aligned}
$$

Definition 2 If there exists $\mathbb{R} \ni \lambda>0$ satisfies the following inequality:

$$
\limsup _{t \rightarrow \infty} \frac{\log \|e(t)\|}{\log \psi(t)} \leqslant-\lambda
$$

where $e(t)=\left(e_{1}^{T}(t), e_{2}^{T}(t), \cdots, e_{M}^{T}(t)\right)^{T}, \psi(t)$ is a $\psi$ type function as defined in Definition 1, then the network (3) is called to be $\psi$-type stable and $\lambda$ is the convergence rate.

Before providing our main results, a lemma needs to be given which plays a key role in our proof. What follows is an assumption for preparing the above mentioned lemma.

Assumption 2 (see [37]) There exist $\eta(t) \in C\left(\mathbb{R}, \mathbb{R}^{+}\right)$ and $\mathbb{R} \ni \sigma>0$ such that:

$$
\sup _{t \in[0, \infty)} \int_{0}^{t} \psi^{\sigma}(h) \eta(h) d h<\infty, \quad \widehat{\psi}(t) \leqslant 1,
$$

in which $\widehat{\psi}(t)$ and $\psi(t)$ are defined in Definition 1. 
Lemma 2 (see [37]) On the premise of Assumption 2, if there exist a differential function $V\left(t, e_{0}(t)\right): \mathbb{R}^{+} \times$ $\mathbb{R}^{n} \rightarrow \mathbb{R}^{+}$and two constants $0<\alpha_{1} \in \mathbb{R}, 0<\alpha_{2} \in \mathbb{R}$ such that

$\left(\alpha_{1}\left\|e_{0}(t)\right\|\right)^{2} \leqslant V\left(t, e_{0}(t)\right)$,

$\left.\dot{V}\left(t, e_{0}(t)\right)\right|_{(3)}+\sigma V\left(t, e_{0}(t)\right) \leqslant \alpha_{2} \eta(t)$,

where $e_{0}(t)$ is a solution of network (3), $\sigma$ and $\eta(t)$ are well defined in Assumption 2, then we said the system

(3) is $\psi$-type stable, namely the drive-response systems (1) and (2) reach general decay anti-synchronization. In addition, the convergence rate is $\frac{\sigma}{2}$.

Remark 2 In recent decades, NNs have been widely applied in various fields, e.g., optimization, parameter estimation, pattern recognition and so on $[4,7,15,26,29]$. As a matter of fact, great majority of these applications depend heavily on the dynamical behaviors of NNs. For example, it is vital that each trajectory of the NNs converges to a unique equilibrium point in order to solve optimization problem by utilizing $\mathrm{NNs}$, that is, the NNs is stable. Hence, many researchers have devoted themselves to studying the stability of NNs [30, $44,46]$. In addition, as pointed out in [14], it is extremely interesting subject to estimate the solution's convergence rate of nonlinear systems, which is prior condition for theoretical analysis and design. However, in many practical cases, the convergence time or speed of the system is difficult to obtain. In 2016, Wang et al. [36] firstly proposed the definition of general decay stability based on $\psi$-type function, also known as $\psi$-type stability. Indeed, when NNs possess $\psi$-type stability, it is helpful to solve the optimization problem and implement content-addressable memories [47]. Note that anti-synchronization is another interesting phenomenon in many applications, such as in chaotic NNs, memristive NNs, and even periodic oscillator $[16,21,24,40]$. In fact, anti-synchronization refers to the disappearance of the sum of the relevant state variables of the nodes in a NN or social network. In above existing literatures [25, 36,37 , the $\psi$-type stability is defined as follows:

$\limsup _{t \rightarrow \infty} \frac{\log \|W(t)-Y(t)\|}{\log \psi(t)} \leqslant-\lambda$.

In Definition 2, the synchronization error $\|W(t)-Y(t)\|$ is changed to $\|W(t)+Y(t)\|$, which means the state variables of two systems (1) and (2) have the same amplitude but the opposite signs. Therefore, the driveresponse systems (1) and (2) can reach decay anti-synchronization if the system (3) is $\psi$-type stable.
In this section, we design the following nonlinear controller for response system (2):

$u_{s}(t)=-q_{s} e_{s}(t)-\beta_{s} \frac{\|e(t)\|^{2} e_{s}(t)}{\|e(t)\|^{2}+\eta(t)}, s=1,2, \cdots, M$,

where $\mathbb{R} \ni q_{s}>0, \mathbb{R} \ni \beta_{s}>0$.

For convenience, we denote $\beta=\max _{1 \leqslant s \leqslant M}\left\{\beta_{s}\right\}, \hat{q}=$ $\operatorname{diag}\left(q_{1}, q_{2}, \cdots, q_{M}\right), \hat{\beta}=\operatorname{diag}\left(\beta_{1}, \beta_{2}, \cdots, \beta_{M}\right)$.

Theorem 1 Under Assumptions 1 and 2, the network(3) is $\psi$-type stable with the convergence rate $\frac{\sigma}{2}$, or the systems (1) and (2) reach general decay anti-synchronization, if

$\Phi_{1}=I_{M} \otimes K_{1}-(\hat{q}+\hat{\beta}) \otimes I_{n}+\sum_{\kappa=1}^{m} c_{\kappa} G^{\kappa} \otimes \Gamma_{\kappa}<0$,

where $K_{1}=-A+\frac{1}{2}\left(B B^{T}+F\right)+\frac{\sigma}{2} I_{n}$.

Proof We construct the following Lyapunov functional for network (3):

$V_{1}(t)=\frac{1}{2} \sum_{s=1}^{M} e_{s}^{T}(t) e_{s}(t)$

It is easy to get $\left(\frac{1}{\sqrt{2}}\|e(t)\|\right)^{2} \leqslant V_{1}(t)$. And one has

$$
\begin{aligned}
\dot{V}_{1}(t)= & \sum_{s=1}^{M} e_{s}^{T}(t)\left(-A e_{s}(t)+B g\left(Y_{s}(t)\right)+B g\left(W_{s}(t)\right)\right. \\
& +\sum_{\kappa=1}^{m} \sum_{j=1}^{M} c_{\kappa} G_{s j}^{\kappa} \Gamma_{\kappa} e_{j}(t)-q_{s} e_{s}(t) \\
& \left.-\beta_{s} \frac{\|e(t)\|^{2} e_{s}(t)}{\|e(t)\|^{2}+\eta(t)}\right) .
\end{aligned}
$$

Obviously,

$$
\begin{aligned}
& e_{s}^{T}(t) B\left(g\left(Y_{s}(t)\right)+g\left(W_{s}(t)\right)\right) \\
\leqslant & \frac{1}{2} e_{s}^{T}(t)\left(B B^{T}+F\right) e_{s}(t) .
\end{aligned}
$$

From (7) and (8), we have

$$
\begin{aligned}
\dot{V}_{1}(t)= & -\sum_{s=1}^{M} \beta_{s} e_{s}^{T}(t) e_{s}(t)+\sum_{s=1}^{M} e_{s}^{T}(t)\left(-A+\frac{1}{2}\left(B B^{T}+F\right)\right. \\
& \left.-q_{s} I_{n}\right) e_{s}(t)-\frac{\|e(t)\|^{2}}{\|e(t)\|^{2}+\eta(t)} \sum_{s=1}^{M} \beta_{s} e_{s}^{T}(t) e_{s}(t) \\
& +\sum_{\kappa=1}^{m} \sum_{s=1}^{M} \sum_{j=1}^{M} c_{\kappa} G_{s j}^{\kappa} e_{s}^{T}(t) \Gamma_{\kappa} e_{j}(t) \\
& +\sum_{s=1}^{M} \beta_{s} e_{s}^{T}(t) e_{s}(t)
\end{aligned}
$$




$$
\begin{aligned}
= & -\sum_{s=1}^{M} \beta_{s} e_{s}^{T}(t) e_{s}(t)+\sum_{s=1}^{M} e_{s}^{T}(t)\left(-A+\frac{1}{2}\left(B B^{T}+F\right)\right. \\
& \left.-q_{s} I_{n}\right) e_{s}(t)+\frac{\eta(t)}{\|e(t)\|^{2}+\eta(t)} \sum_{s=1}^{M} \beta_{s} e_{s}^{T}(t) e_{s}(t) \\
& +\sum_{\kappa=1}^{m} \sum_{s=1}^{M} \sum_{j=1}^{M} c_{\kappa} G_{s j}^{\kappa} e_{s}^{T}(t) \Gamma_{\kappa} e_{j}(t) \\
\leqslant & \frac{\beta\|e(t)\|^{2} \eta(t)}{\|e(t)\|^{2}+\eta(t)}+\sum_{s=1}^{M} e_{s}^{T}(t)\left(-A+\frac{1}{2}\left(B B^{T}+F\right)-q_{s} I_{n}\right. \\
& \left.-\beta_{s} I_{n}\right) e_{s}(t)+\sum_{\kappa=1}^{m} \sum_{s=1}^{M} \sum_{j=1}^{M} c_{\kappa} G_{s j}^{\kappa} e_{s}^{T}(t) \Gamma_{\kappa} e_{j}(t) .
\end{aligned}
$$

3.2 General decay anti-synchronization of MWCNNs with delayed coupling

In this section, a MWCNN model with delayed coupling is considered which can be represented as follows:

$$
\begin{aligned}
\dot{Y}_{s}(t)= & -A Y_{s}(t)+B g\left(Y_{s}(t)\right)+\sum_{\kappa=1}^{m} \sum_{j=1}^{M} c_{\kappa} G_{s j}^{\kappa} \Gamma_{\kappa} Y_{j}(t \\
& \left.-\tau_{\kappa}(t)\right), s=1,2, \cdots, M
\end{aligned}
$$

where $Y_{s}(t), g(\cdot), A, B, c_{\kappa}, G_{s j}^{\kappa}, \Gamma_{\kappa}$ are defined similarly as those in Section 3.1, and the time-varying delay $\tau_{\kappa}(t)(\kappa=1,2, \cdots, m)$ satisfies $0 \leqslant \tau_{\kappa}(t) \leqslant \tau$.

Consider the network model (11) as the drive system. Then its corresponding response system is introduced as:

Combine (6) with (9), one has

$$
\begin{aligned}
& \dot{V}_{1}(t)+\sigma V_{1}(t) \\
\leqslant & \frac{\beta\|e(t)\|^{2} \eta(t)}{\|e(t)\|^{2}+\eta(t)}+\sum_{s=1}^{M} e_{s}^{T}(t)\left(-A+\frac{1}{2}\left(B B^{T}+F\right)\right. \\
& \left.-q_{s} I_{n}-\beta_{s} I_{n}\right) e_{s}(t)+\frac{\sigma}{2} \sum_{s=1}^{M} e_{s}^{T}(t) e_{s}(t) \\
& +\sum_{\kappa=1}^{m} \sum_{s=1}^{M} \sum_{j=1}^{M} c_{\kappa} G_{s j}^{\kappa} e_{s}^{T}(t) \Gamma_{\kappa} e_{j}(t) \\
\leqslant & \sum_{s=1}^{M} e_{s}^{T}(t)\left(-A+\frac{1}{2}\left(B B^{T}+F\right)-q_{s} I_{n}-\beta_{s} I_{n}\right. \\
& \left.+\frac{\sigma}{2} I_{n}\right) e_{s}(t)+\sum_{\kappa=1}^{m} \sum_{s=1}^{M} \sum_{j=1}^{M} c_{\kappa} G_{s j}^{\kappa} e_{s}^{T}(t) \Gamma_{\kappa} e_{j}(t) \\
& +\beta \eta(t) \\
= & \beta \eta(t)+e^{T}(t)\left[I_{M} \otimes\left(-A+\frac{1}{2}\left(B B^{T}+F\right)+\frac{\sigma}{2} I_{n}\right)\right. \\
& \left.-(\hat{q}+\hat{\beta}) \otimes I_{n}+\sum_{\kappa=1}^{m} c_{\kappa} G^{\kappa} \otimes \Gamma_{\kappa}\right] e(t),
\end{aligned}
$$

where $e(t)=\left(e_{1}^{T}(t), e_{2}^{T}(t), \cdots, e_{M}^{T}(t)\right)^{T}$. According to $(5)$, one has

$$
\dot{V}_{1}(t)+\sigma V_{1}(t) \leqslant \beta \eta(t)
$$

Take $\alpha_{1}=\frac{1}{\sqrt{2}}, \alpha_{2}=\beta$, then it is easy to obtain the network (3) is $\psi$-type stable with convergence rate $\frac{\sigma}{2}$. In other words, the drive system (1) and response system (2) reach general decay anti-synchronization.

$$
\begin{aligned}
\dot{W}_{s}(t)= & -A W_{s}(t)+B g\left(W_{s}(t)\right)+\sum_{\kappa=1}^{m} \sum_{j=1}^{M} c_{\kappa} G_{s j}^{\kappa} \Gamma_{\kappa} W_{j}(t \\
& \left.-\tau_{\kappa}(t)\right)+u_{s}(t)
\end{aligned}
$$

where $s=1,2, \cdots, M ; W_{s}(t), A, B, c_{\kappa}, G_{s j}^{\kappa}, \Gamma_{\kappa}, u_{s}(t)$ have the same definitions as in system $(2) ; \tau_{\kappa}(t)(\kappa=$ $1,2, \cdots, m)$ has the same definition as in system (11).

Take $e_{s}(t)=Y_{s}(t)+W_{s}(t)$. By (11) and (12), we can obtain

$$
\begin{aligned}
\dot{e}_{s}(t)= & -A e_{s}(t)+B g\left(W_{s}(t)\right)+B g\left(Y_{s}(t)\right)+u_{s}(t) \\
& +\sum_{\kappa=1}^{m} \sum_{j=1}^{M} c_{\kappa} G_{s j}^{\kappa} \Gamma_{\kappa} e_{j}\left(t-\tau_{\kappa}(t)\right) .
\end{aligned}
$$

We construct the same nonlinear controller (4) for response system (12) in this section.

Theorem 2 Suppose $\dot{\tau}_{\kappa}(t) \leqslant \gamma_{\kappa}<1$. Under Assumptions 1 and 2, the network (13) is $\psi$-type stable with the convergence rate $\frac{\sigma}{2}$, or the systems (11) and (12) reach general decay anti-synchronization, if

$$
\begin{aligned}
\Phi_{2}= & I_{M} \otimes K_{2}+\frac{1}{2} \sum_{\kappa=1}^{m} c_{\kappa}\left(G^{\kappa}\right)^{2} \otimes\left(\Gamma_{\kappa}\right)^{2}-(\hat{q} \\
& +\hat{\beta}) \otimes I_{n}<0, \\
\Lambda_{2}= & \tau \sigma+\frac{\sigma}{2} \sum_{\kappa=1}^{m} \frac{c_{\kappa}}{1-\gamma_{\kappa}}<1,
\end{aligned}
$$

where $K_{2}=-A+\frac{1}{2}\left(B B^{T}+F\right)+\left(\frac{\sigma}{2}+\tau+\frac{1}{2} \sum_{\kappa=1}^{m} \frac{c_{\kappa}}{1-\gamma_{\kappa}}\right) I_{n}$.

Proof Define the following Lyapunov functional for network (13):

$V_{2}(t)=\frac{1}{2} \sum_{s=1}^{M} e_{s}^{T}(t) e_{s}(t)+\frac{1}{2} \sum_{\kappa=1}^{m} \frac{c_{\kappa}}{1-\gamma_{\kappa}} \int_{t-\tau_{\kappa}(t)}^{t} e^{T}(h) e(h) d h$ 


$$
+\int_{-\tau}^{0} \int_{t+\rho}^{t} e^{T}(h) e(h) d h d \rho .
$$

Obviously, $\left(\frac{1}{\sqrt{2}}\|e(t)\|\right)^{2} \leqslant V_{2}(t)$ and we can deduce from (16) as follows:

$$
\begin{aligned}
V_{2}(t) \leqslant & \frac{1}{2} \sum_{s=1}^{M} e_{s}^{T}(t) e_{s}(t)+\frac{1}{2} \sum_{\kappa=1}^{m} \frac{c_{\kappa}}{1-\gamma_{\kappa}} \int_{t-\tau}^{t} e^{T}(h) e(h) d h \\
& +\tau \int_{t-\tau}^{t} e^{T}(h) e(h) d h \\
= & \left(\tau+\frac{1}{2} \sum_{\kappa=1}^{m} \frac{c_{\kappa}}{1-\gamma_{\kappa}}\right) \int_{t-\tau}^{t} e^{T}(h) e(h) d h \\
& +\frac{1}{2} \sum_{s=1}^{M} e_{s}^{T}(t) e_{s}(t)
\end{aligned}
$$

By calculating the derivative of (16), one has

$$
\begin{aligned}
\dot{V}_{2}(t) \leqslant & \sum_{s=1}^{M} e_{s}^{T}(t)\left(-A e_{s}(t)+\sum_{\kappa=1}^{m} \sum_{j=1}^{M} c_{\kappa} G_{s j}^{\kappa} \Gamma_{\kappa} e_{j}\left(t-\tau_{\kappa}(t)\right)\right. \\
& +B g\left(Y_{s}(t)\right)+B g\left(W_{s}(t)\right)-\beta_{s} \frac{\|e(t)\|^{2} e_{s}(t)}{\|e(t)\|^{2}+\eta(t)} \\
& \left.-q_{s} e_{s}(t)\right)+\tau e^{T}(t) e(t)-\int_{t-\tau}^{t} e^{T}(h) e(h) d h \\
& +\frac{1}{2} \sum_{\kappa=1}^{m} \frac{c_{\kappa}}{1-\gamma_{\kappa}} e^{T}(t) e(t)-\frac{1}{2} \sum_{\kappa=1}^{m} c_{\kappa} e^{T}(t \\
& \left.-\tau_{\kappa}(t)\right) e\left(t-\tau_{\kappa}(t)\right)
\end{aligned}
$$

where $e\left(t-\tau_{\kappa}(t)\right)=\left(e_{1}^{T}\left(t-\tau_{\kappa}(t)\right), e_{2}^{T}\left(t-\tau_{\kappa}(t)\right), \cdots, e_{M}^{T}(t-\right.$ $\left.\left.\tau_{\kappa}(t)\right)\right)^{T}$. Obviously,

$$
\begin{aligned}
& \sum_{\kappa=1}^{m} \sum_{s=1}^{M} \sum_{j=1}^{M} c_{\kappa} G_{s j}^{\kappa} e_{s}^{T}(t) \Gamma_{\kappa} e_{j}\left(t-\tau_{\kappa}(t)\right) \\
\leqslant & \frac{1}{2} \sum_{\kappa=1}^{m} c_{\kappa} e^{T}(t)\left(\left(G^{\kappa}\right)^{2} \otimes\left(\Gamma_{\kappa}\right)^{2}\right) e(t) \\
& +\frac{1}{2} \sum_{\kappa=1}^{m} c_{\kappa} e^{T}\left(t-\tau_{\kappa}(t)\right) e\left(t-\tau_{\kappa}(t)\right) .
\end{aligned}
$$

Combine (18) with (19), one has

$$
\begin{aligned}
\dot{V}_{2}(t) \leqslant & \sum_{s=1}^{M} e_{s}^{T}(t)\left(-A e_{s}(t)+B g\left(Y_{s}(t)\right)-\beta_{s} \frac{\|e(t)\|^{2} e_{s}(t)}{\|e(t)\|^{2}+\eta(t)}\right. \\
& \left.+B g\left(W_{s}(t)\right)-q_{s} e_{s}(t)\right)+\frac{1}{2} \sum_{\kappa=1}^{m} \frac{c_{\kappa}}{1-\gamma_{\kappa}} e^{T}(t) e(t) \\
& +\frac{1}{2} \sum_{\kappa=1}^{m} c_{\kappa} e^{T}(t)\left(\left(G^{\kappa}\right)^{2} \otimes\left(\Gamma_{\kappa}\right)^{2}\right) e(t) \\
& +\tau e^{T}(t) e(t)-\int_{t-\tau}^{t} e^{T}(h) e(h) d h
\end{aligned}
$$

$$
\begin{aligned}
\leqslant & \sum_{s=1}^{M} \beta_{s} e_{s}^{T}(t) e_{s}(t)+\tau e^{T}(t) e(t)+\sum_{s=1}^{M} e_{s}^{T}(t)\left(-A+\frac{1}{2}\left(B B^{T}\right.\right. \\
& \left.+F)-q_{s} I_{n}\right) e_{s}(t)+\frac{1}{2} \sum_{\kappa=1}^{m} c_{\kappa} e^{T}(t)\left(\left(G^{\kappa}\right)^{2} \otimes\left(\Gamma_{\kappa}\right)^{2}\right) e(t) \\
& -\frac{\|e(t)\|^{2}}{\|e(t)\|^{2}+\eta(t)} \sum_{s=1}^{M} \beta_{s} e_{s}^{T}(t) e_{s}(t)-\sum_{s=1}^{M} \beta_{s} e_{s}^{T}(t) e_{s}(t) \\
& +\frac{1}{2} \sum_{\kappa=1}^{m} \frac{c_{\kappa}}{1-\gamma_{\kappa}} e^{T}(t) e(t)-\int_{t-\tau}^{t} e^{T}(h) e(h) d h \\
\leqslant & \frac{\beta\|e(t)\|^{2} \eta(t)}{\|e(t)\|^{2}+\eta(t)}+\sum_{s=1}^{M} e_{s}^{T}(t)\left(-A+\frac{1}{2}\left(B B^{T}+F\right)-q_{s} I_{n}\right. \\
& \left.-\beta_{s} I_{n}\right) e_{s}(t)+\frac{1}{2} \sum_{\kappa=1}^{m} c_{\kappa} e^{T}(t)\left(\left(G^{\kappa}\right)^{2} \otimes\left(\Gamma_{\kappa}\right)^{2}\right) e(t) \\
& +\tau e^{T}(t) e(t)-\int_{t-\tau}^{t} e^{T}(h) e(h) d h \\
& +\frac{1}{2} \sum_{\kappa=1}^{m} \frac{c_{\kappa}}{1-\gamma_{\kappa}} e^{T}(t) e(t) .
\end{aligned}
$$

Combine (17) with (20), we have

$$
\text { s.t. }
$$




$$
\begin{aligned}
& \left.+\left(\frac{\sigma}{2}+\frac{1}{2} \sum_{\kappa=1}^{m} \frac{c_{\kappa}}{1-\gamma_{\kappa}}+\tau\right) I_{n}\right)-(\hat{q}+\hat{\beta}) \otimes I_{n} \\
& \left.+\frac{1}{2} \sum_{\kappa=1}^{m} c_{\kappa}\left(G^{\kappa}\right)^{2} \otimes\left(\Gamma_{\kappa}\right)^{2}\right] e(t)+(\tau \sigma-1 \\
& \left.+\frac{\sigma}{2} \sum_{\kappa=1}^{m} \frac{c_{\kappa}}{1-\gamma_{\kappa}}\right) \int_{t-\tau}^{t} e^{T}(h) e(h) d h .
\end{aligned}
$$

by the partial differential equations as follows:

$$
\begin{aligned}
\frac{\partial Y_{s}(\vartheta, t)}{\partial t}= & -A Y_{s}(\vartheta, t)+D \triangle Y_{s}(\vartheta, t)+B g\left(Y_{s}(\vartheta, t)\right) \\
& +\sum_{\kappa=1}^{m} \sum_{j=1}^{M} c_{\kappa} G_{s j}^{\kappa} \Gamma_{\kappa} Y_{j}(\vartheta, t)
\end{aligned}
$$

According to (14) and (15), we can obtain

$$
\dot{V}_{2}(t)+\sigma V_{2}(t) \leqslant \beta \eta(t) \text {. }
$$

Take $\alpha_{1}=\frac{1}{\sqrt{2}}, \alpha_{2}=\beta$, it is easy to obtain that network (13) is $\psi$-type stable with convergence rate $\frac{\sigma}{2}$. Then, the drive system (11) and response system (12) reach general decay anti-synchronization.

Remark 3 Recently, some scholars investigated the synchronization and finite-time synchronization of NNs by using integrating inequality technique, instead of conventional methods, such as linear matrix inequality method, matrix measure strategy and so on [50-52]. In [50], Zhang et al. established some new sufficient conditions on global asymptotic synchronization for delayed inertial NNs by constructing different Lyapunov functions and using the constructed integral inequality. These results on the synchronization are novel and interesting. It is noteworthy that the network models in the above mentioned literatures are single-weighted. Actually, numerous existing networks can be represented more precisely by complex networks with multiple weights [2, $3,54,55]$. In this section, we derive two sufficient conditions for reaching decay anti-synchronization of the considered MWCNNs by designing appropriate nonlinear controller and using Lyapunov functional method as well as commonly used inequality techniques. To the best of our knowledge, this is the first paper toward to studying the decay anti-synchronization of MWCNNs. In our future work, it would be very interesting to study the decay anti-synchronization of MWCNNs by using some novel inequality techniques, e.g., integral inequality constructed in Lemma 2.1 of [50].

\section{General decay anti-synchronization of MWCRDNNs with and without delayed coupling}

\subsection{General decay anti-synchronization of MWCRDNNs}

In this section, the model of MWCNNs with reactiondiffusion terms is considered which can be characterized

where $s=1,2, \cdots, M, Y_{s}(\vartheta, t)=\left(Y_{s 1}(\vartheta, t), Y_{s 2}(\vartheta, t), \cdots\right.$ ,$\left.Y_{s n}(\vartheta, t)\right)^{T} \in \mathbb{R}^{n}$ is the state vector of the $s$ th neuron at time $t$ and in space $\vartheta ; \vartheta=\left(\vartheta_{1}, \vartheta_{2}, \cdots, \vartheta_{p}\right)^{T} \in \Theta \subset$ $\mathbb{R}^{p} ; D=\operatorname{diag}\left(d_{1}, d_{2}, \cdots, d_{n}\right), d_{s}>0$ is the transmission diffusion coefficient; $g\left(Y_{s}(\vartheta, t)\right)=\left(g_{1}\left(Y_{s 1}(\vartheta, t)\right), g_{2}(\right.$ $\left.\left.Y_{s 2}(\vartheta, t)\right), \cdots, g_{n}\left(Y_{s n}(\vartheta, t)\right)\right)^{T} \in \mathbb{R}^{n} ; \Delta=\sum_{r=1}^{p} \frac{\partial^{2}}{\partial \vartheta_{r}^{2}}$ symbols the Laplace diffusion operator on $\Theta ; A, B, c_{\kappa}, \Gamma_{\kappa}$, $G_{s j}^{\kappa}$ have the same meanings as in Section 3.1.

For the network (21),

$$
\begin{aligned}
& Y_{s}(\vartheta, 0)=\phi_{s}(\vartheta) \in \mathbb{R}^{n}, \quad \vartheta \in \Theta, \\
& Y_{s}(\vartheta, t)=0, \quad(\vartheta, t) \in \partial \Theta \times[0,+\infty) .
\end{aligned}
$$

Consider the network model (21) as the drive system. Then its corresponding response system is introduced as:

$$
\begin{aligned}
\frac{\partial W_{s}(\vartheta, t)}{\partial t}= & -A W_{s}(\vartheta, t)+D \triangle W_{s}(\vartheta, t)+B g\left(W_{s}(\vartheta, t)\right) \\
& +\sum_{\kappa=1}^{m} \sum_{j=1}^{M} c_{\kappa} G_{s j}^{\kappa} \Gamma_{\kappa} W_{j}(\vartheta, t)+u_{s}(\vartheta, t), \quad(22)
\end{aligned}
$$

where $s=1,2, \cdots, M, W_{s}(\vartheta, t)=\left(W_{s 1}(\vartheta, t), W_{s 2}(\vartheta, t)\right.$, $\left.\cdots, W_{s n}(\vartheta, t)\right)^{T} \in \mathbb{R}^{n}$ is the state vector of the $s$ th neuron at time $t$ and in space $\vartheta ; u_{s}(\vartheta, t)=\left(u_{s 1}(\vartheta, t), u_{s 2}(\vartheta, t)\right.$ $\left., \cdots, u_{s n}(\vartheta, t)\right)^{T} \in \mathbb{R}^{n}$ is the suitable controller to achieve a certain control objective; $A, B, D, c_{\kappa}, G_{s j}^{\kappa}, \Gamma_{\kappa}$ have the same definitions as in system (21).

For the network (22),

$$
\begin{aligned}
& W_{s}(\vartheta, 0)=\varphi_{s}(\vartheta) \in \mathbb{R}^{n}, \quad \vartheta \in \Theta, \\
& W_{s}(\vartheta, t)=0, \quad(\vartheta, t) \in \partial \Theta \times[0,+\infty) .
\end{aligned}
$$

Take $e_{s}(\vartheta, t)=Y_{s}(\vartheta, t)+W_{s}(\vartheta, t)$. By $(21)$ and $(22)$, it is easy to get

$$
\begin{aligned}
\frac{\partial e_{s}(\vartheta, t)}{\partial t}= & -A e_{s}(\vartheta, t)+D \triangle e_{s}(\vartheta, t)+B g\left(Y_{s}(\vartheta, t)\right) \\
& +B g\left(W_{s}(\vartheta, t)\right)+\sum_{\kappa=1}^{m} \sum_{j=1}^{M} c_{\kappa} G_{s j}^{\kappa} \Gamma_{\kappa} e_{j}(\vartheta, t) \\
& +u_{s}(\vartheta, t) .
\end{aligned}
$$

Definition 3 If there exists a constant $\mathbb{R} \ni \lambda>0$ such that

$\limsup _{t \rightarrow \infty} \frac{\log \|e(\cdot, t)\|}{\log \psi(t)} \leqslant-\lambda$, 
where $e(\vartheta, t)=\left(e_{1}^{T}(\vartheta, t), e_{2}^{T}(\vartheta, t), \cdots, e_{M}^{T}(\vartheta, t)\right)^{T}, \psi(t)$ is a $\psi$-type function as defined in Definition 1 , then the network (23) is called to be $\psi$-type stable, where $\lambda$ is the convergence rate when $e(\vartheta, t) \rightarrow 0$.

For convenience, we denote

$$
\begin{aligned}
Y(\vartheta, t) & =\left(Y_{1}^{T}(\vartheta, t), Y_{2}^{T}(\vartheta, t), \cdots, Y_{M}^{T}(\vartheta, t)\right)^{T}, \\
W(\vartheta, t) & =\left(W_{1}^{T}(\vartheta, t), W_{2}^{T}(\vartheta, t), \cdots, W_{M}^{T}(\vartheta, t)\right)^{T},
\end{aligned}
$$
$\hat{g}(Y(\vartheta, t))=\left(g^{T}\left(Y_{1}(\vartheta, t)\right), g^{T}\left(Y_{2}(\vartheta, t)\right), \cdots, g^{T}\left(Y_{M}(\vartheta, t)\right)\right)^{T}$, $\hat{g}(W(\vartheta, t))=\left(g^{T}\left(W_{1}(\vartheta, t)\right), g^{T}\left(W_{2}(\vartheta, t)\right), \cdots, g^{T}\left(W_{M}(\vartheta, t)\right)\right)^{T}$.

For response system (22), the following nonlinear controller is designed :

$u_{s}(\vartheta, t)=-q_{s} e_{s}(\vartheta, t)-\beta_{s} \frac{\|e(\cdot, t)\|^{2} e_{s}(\vartheta, t)}{\|e(\cdot, t)\|^{2}+\eta(t)}$,

where $s=1,2, \cdots, M, \mathbb{R} \ni q_{s}>0, \mathbb{R} \ni \beta_{s}>0$.

For convenience, we denote $\beta=\max _{1 \leqslant s \leqslant M}\left\{\beta_{s}\right\}, \hat{q}=$ $\operatorname{diag}\left(q_{1}, q_{2}, \cdots, q_{M}\right), \hat{\beta}=\operatorname{diag}\left(\beta_{1}, \beta_{2}, \cdots, \beta_{M}\right)$.

Theorem 3 Under Assumptions 1 and 2, the network (23) is $\psi$-type stable with the convergence rate $\frac{\sigma}{2}$, or the systems (21)and (22) achieve general decay antisynchronization, if

$$
\begin{aligned}
\Phi_{3}= & I_{M} \otimes\left(K_{1}-\sum_{r=1}^{p} \frac{1}{\iota_{r}^{2}} D\right)-(\hat{q}+\hat{\beta}) \otimes I_{n} \\
& +\sum_{\kappa=1}^{m} c_{\kappa} G^{\kappa} \otimes \Gamma_{\kappa}<0 .
\end{aligned}
$$

Proof Construct the following Lyapunov functional for network (23):

$V_{3}(t)=\frac{1}{2} \sum_{s=1}^{M} \int_{\Theta} e_{s}^{T}(\vartheta, t) e_{s}(\vartheta, t) d \vartheta$

Obviously, $\left(\frac{1}{\sqrt{2}}\|e(\cdot, t)\|\right)^{2} \leqslant V_{3}(t)$. And, one has

$$
\begin{aligned}
\dot{V}_{3}(t)= & \sum_{s=1}^{M} \int_{\Theta} e_{s}^{T}(\vartheta, t) \frac{\partial e_{s}(\vartheta, t)}{\partial t} d \vartheta \\
= & \sum_{s=1}^{M} \int_{\Theta} e_{s}^{T}(\vartheta, t)\left(-A e_{s}(\vartheta, t)+B g\left(Y_{s}(\vartheta, t)\right)\right. \\
& +D \triangle e_{s}(\vartheta, t)+B g\left(W_{s}(\vartheta, t)\right)-\beta_{s} \frac{\|e(\cdot, t)\|^{2} e_{s}(\vartheta, t)}{\|e(\cdot, t)\|^{2}+\eta(t)} \\
& \left.-q_{s} e_{s}(\vartheta, t)+\sum_{\kappa=1}^{m} \sum_{j=1}^{M} c_{\kappa} G_{s j}^{\kappa} \Gamma_{\kappa} e_{j}(\vartheta, t)\right) d \vartheta \\
& +\sum_{s=1}^{M} \beta_{s} \int_{\Theta} e_{s}^{T}(\vartheta, t) e_{s}(\vartheta, t) d \vartheta
\end{aligned}
$$

$$
\begin{aligned}
& -\sum_{s=1}^{M} \beta_{s} \int_{\Theta} e_{s}^{T}(\vartheta, t) e_{s}(\vartheta, t) d \vartheta \\
= & \int_{\Theta} e^{T}(\vartheta, t)\left(I_{M} \otimes B\right)(\hat{g}(Y(\vartheta, t))+\hat{g}(W(\vartheta, t))) d \vartheta \\
& +\int_{\Theta} e^{T}(\vartheta, t)\left(\sum_{\kappa=1}^{m} c_{\kappa} G^{\kappa} \otimes \Gamma_{\kappa}-I_{M} \otimes A-(\hat{\beta}\right. \\
& \left.+\hat{q}) \otimes I_{n}\right) e(\vartheta, t) d \vartheta+\int_{\Theta} e^{T}(\vartheta, t)\left(I_{M} \otimes D\right) \triangle e(\vartheta, t) d \vartheta \\
& +\frac{\eta(t)}{\|e(\cdot, t)\|^{2}+\eta(t)} \sum_{s=1}^{M} \beta_{s} \int_{\Theta} e_{s}^{T}(\vartheta, t) e_{s}(\vartheta, t) d \vartheta \\
\leqslant & \int_{\Theta} e^{T}(\vartheta, t)\left(I_{M} \otimes B\right)(\hat{g}(Y(\vartheta, t))+\hat{g}(W(\vartheta, t))) d \vartheta \\
& +\int_{\Theta} e^{T}(\vartheta, t)\left(\sum_{\kappa=1}^{m} c_{\kappa} G^{\kappa} \otimes \Gamma_{\kappa}-I_{M} \otimes A-(\hat{\beta}\right. \\
& \left.+\hat{q}) \otimes I_{n}\right) e(\vartheta, t) d \vartheta+\frac{\beta\|e(\cdot, t)\|^{2} \eta(t)}{\|e(\cdot, t)\|^{2}+\eta(t)} \\
& +\int_{\Theta} e^{T}(\vartheta, t)\left(I_{M} \otimes D\right) \triangle e(\vartheta, t) d \vartheta
\end{aligned}
$$

where $e(\vartheta, t)=\left(e_{1}^{T}(\vartheta, t), e_{2}^{T}(\vartheta, t), \cdots, e_{M}^{T}(\vartheta, t)\right)^{T}$. From the Green's formula, one has

$\int_{\Theta} e_{s l}(\vartheta, t) \triangle e_{s j}(\vartheta, t) d \vartheta=-\sum_{r=1}^{p} \int_{\Theta} \frac{\partial e_{s l}(\vartheta, t)}{\partial \vartheta_{r}} \frac{\partial e_{s j}(\vartheta, t)}{\partial \vartheta_{r}} d \vartheta$

where $l, j \in\{1,2, \cdots, n\}, s=1,2, \cdots, M$. Let $\pi(\vartheta, t)=$ $\left(I_{M} \otimes \sqrt{D}\right) e(\vartheta, t)$. From Lemma 1 , we can obtain

$$
\begin{aligned}
& \int_{\Theta} e^{T}(\vartheta, t)\left(I_{M} \otimes D\right) \triangle e(\vartheta, t) d \vartheta \\
= & -\sum_{r=1}^{p} \sum_{s=1}^{M} \sum_{j=1}^{n} \sum_{l=1}^{n} d_{l} \int_{\Theta} \frac{\partial e_{s j}(\vartheta, t)}{\partial \vartheta_{r}} \frac{\partial e_{s l}(\vartheta, t)}{\partial \vartheta_{r}} d \vartheta \\
= & -\sum_{r=1}^{p} \int_{\Theta}\left(\frac{\partial e(\vartheta, t)}{\partial \vartheta_{r}}\right)^{T}\left(I_{M} \otimes D\right) \frac{\partial e(\vartheta, t)}{\partial \vartheta_{r}} d \vartheta \\
= & -\sum_{r=1}^{p} \int_{\Theta}\left(\frac{\partial \pi(\vartheta, t)}{\partial \vartheta_{r}}\right)^{T} \frac{\partial \pi(\vartheta, t)}{\partial \vartheta_{r}} d \vartheta \\
\leqslant & -\sum_{r=1}^{p} \frac{1}{\iota_{r}^{2}} \int_{\Theta} \pi^{T}(\vartheta, t) \pi(\vartheta, t) d \vartheta \\
= & -\sum_{r=1}^{p} \frac{1}{\iota_{r}^{2}} \int_{\Theta} e^{T}(\vartheta, t)\left(I_{M} \otimes D\right) e(\vartheta, t) d \vartheta .
\end{aligned}
$$

Furthermore, one can easily deduce

$$
\begin{aligned}
& e^{T}(\vartheta, t)\left(I_{M} \otimes B\right)(\hat{g}(Y(\vartheta, t))+\hat{g}(W(\vartheta, t)) \\
\leqslant & \frac{1}{2} e^{T}(\vartheta, t)\left(I_{M} \otimes\left(B B^{T}+F\right)\right) e(\vartheta, t) .
\end{aligned}
$$


From (27) to (29), we can obtain

$$
\begin{aligned}
\dot{V}_{3}(t) \leqslant & \int_{\Theta} e^{T}(\vartheta, t)\left[I_{M} \otimes\left(-A+\frac{1}{2}\left(B B^{T}+F\right)-\sum_{r=1}^{p} \frac{1}{\iota_{r}^{2}} D\right)\right. \\
& \left.-(\hat{\beta}+\hat{q}) \otimes I_{n}+\sum_{\kappa=1}^{m} c_{\kappa} G^{\kappa} \otimes \Gamma_{\kappa}\right] e(\vartheta, t) d \vartheta \\
& +\frac{\beta\|e(\cdot, t)\|^{2} \eta(t)}{\|e(\cdot, t)\|^{2}+\eta(t)}
\end{aligned}
$$

Combine (26) with (30), we have

$$
\begin{aligned}
\dot{V}_{3}(t)+\sigma V_{3}(t) \leqslant & \int_{\Theta} e^{T}(\vartheta, t)\left[I _ { M } \otimes \left(-A+\frac{1}{2}\left(B B^{T}+F\right)\right.\right. \\
& \left.-\sum_{r=1}^{p} \frac{1}{\iota_{r}^{2}} D\right)+\sum_{\kappa=1}^{m} c_{\kappa} G^{\kappa} \otimes \Gamma_{\kappa}-(\hat{\beta} \\
& \left.+\hat{q}) \otimes I_{n}\right] e(\vartheta, t) d \vartheta+\frac{\beta\|e(\cdot, t)\|^{2} \eta(t)}{\|e(\cdot, t)\|^{2}+\eta(t)} \\
& +\frac{\sigma}{2} \sum_{s=1}^{M} \int_{\Theta} e_{s}^{T}(\vartheta, t) e_{s}(\vartheta, t) d \vartheta \\
\leqslant & \int_{\Theta} e^{T}(\vartheta, t)\left[I _ { M } \otimes \left(-A+\frac{1}{2}\left(B B^{T}+F\right)\right.\right. \\
& \left.-\sum_{r=1}^{p} \frac{1}{\iota_{r}^{2}} D+\frac{\sigma}{2} I_{n}\right)-(\hat{\beta}+\hat{q}) \otimes I_{n} \\
& \left.+\sum_{\kappa=1}^{m} c_{\kappa} G^{\kappa} \otimes \Gamma_{\kappa}\right] e(\vartheta, t) d \vartheta+\beta \eta(t) .
\end{aligned}
$$

According to (25), one has

$$
\dot{V}_{3}(t)+\sigma V_{3}(t) \leqslant \beta \eta(t) .
$$

Take $\alpha_{1}=\frac{1}{\sqrt{2}}, \alpha_{2}=\beta$, it is easy to obtain that network (23) is $\psi$-type stable with convergence rate $\frac{\sigma}{2}$. Then, the drive system (21) and response system (22) reach general decay anti-synchronization.

\subsection{General decay anti-synchronization of} MWCRDNNs with delayed coupling

In this section, the following MWCRDNNs model with delayed coupling is considered:

$$
\begin{aligned}
\frac{\partial Y_{s}(\vartheta, t)}{\partial t}= & -A Y_{s}(\vartheta, t)+D \triangle Y_{s}(\vartheta, t)+B g\left(Y_{s}(x, t)\right) \\
& +\sum_{\kappa=1}^{m} \sum_{j=1}^{M} c_{\kappa} G_{s j}^{\kappa} \Gamma_{\kappa} Y_{j}\left(\vartheta, t-\tau_{\kappa}(t)\right)
\end{aligned}
$$

where $s=1,2, \cdots, M, Y_{s}(\vartheta, t), g(\cdot), A, B, D, c_{\kappa}, G_{s j}^{\kappa}$, $\Gamma_{\kappa}$ are defined similarly as those in Section 4.1, the time-varying delay $\tau_{\kappa}(t)(\kappa=1,2, \cdots, m)$ satisfies $0 \leqslant$ $\tau_{\kappa}(t) \leqslant \tau$.
For the network (31),

$Y_{s}(\vartheta, t)=\phi_{s}(\vartheta, t), \quad \vartheta \in \Theta \times[-\tau, 0]$
$Y_{s}(\vartheta, t)=0, \quad(\vartheta, t) \in \partial \Theta \times[-\tau,+\infty)$

Consider the network model (31) as the drive system. Then its corresponding response system is introduced as:

$$
\begin{aligned}
\frac{\partial W_{s}(\vartheta, t)}{\partial t}= & -A W_{s}(\vartheta, t)+D \triangle W_{s}(\vartheta, t)+B g\left(W_{s}(\vartheta, t)\right) \\
& +u_{s}(\vartheta, t)+\sum_{\kappa=1}^{m} \sum_{j=1}^{M} c_{\kappa} G_{s j}^{\kappa} \Gamma_{\kappa} W_{j}(\vartheta, t \\
& \left.-\tau_{\kappa}(t)\right), \quad s=1,2, \cdots, M
\end{aligned}
$$

where $A, B, D, c_{\kappa}, G_{s j}^{\kappa}, \Gamma_{\kappa}, W_{s}(\vartheta, t), u_{s}(\vartheta, t), \tau_{\kappa}(t)$ represent the same meanings as in system (31).

For the network (32),

$W_{s}(\vartheta, t)=\varphi_{s}(\vartheta, t), \quad \vartheta \in \Theta \times[-\tau, 0]$, $W_{s}(\vartheta, t)=0, \quad(\vartheta, t) \in \partial \Theta \times[-\tau,+\infty)$.

Take $e_{s}(\vartheta, t)=Y_{s}(\vartheta, t)+W_{s}(\vartheta, t)$. By $(31)$ and $(32)$, we can obtain

$$
\begin{aligned}
\frac{\partial e_{s}(\vartheta, t)}{\partial t}= & -A e_{s}(\vartheta, t)+D \triangle e_{s}(\vartheta, t)+B g\left(Y_{s}(\vartheta, t)\right) \\
& +\sum_{\kappa=1}^{m} \sum_{j=1}^{M} c_{\kappa} G_{s j}^{\kappa} \Gamma_{\kappa} e_{j}\left(\vartheta, t-\tau_{\kappa}(t)\right) \\
& +B g\left(W_{s}(\vartheta, t)\right)+u_{s}(\vartheta, t)
\end{aligned}
$$

We construct the same nonlinear controller (24) for response system (32) in this section.

Theorem 4 Suppose $\dot{\tau}_{\kappa}(t) \leqslant \gamma_{\kappa}<1$. Under Assumptions 1 and 2, the network (33) is $\psi$-type stable with the convergence rate $\frac{\sigma}{2}$, or the systems (31) and (32) reach general decay anti-synchronization, if

$$
\begin{aligned}
\Phi_{4}= & I_{M} \otimes\left(K_{2}-\sum_{r=1}^{p} \frac{1}{\iota_{r}^{2}} D\right)+\frac{1}{2} \sum_{\kappa=1}^{m} c_{\kappa}\left(G^{\kappa}\right)^{2} \otimes\left(\Gamma_{\kappa}\right)^{2} \\
& -(\hat{q}+\hat{\beta}) \otimes I_{n}<0 \\
\Lambda_{4}= & \tau \sigma+\frac{\sigma}{2} \sum_{\kappa=1}^{m} \frac{c_{\kappa}}{1-\gamma_{\kappa}}<1 .
\end{aligned}
$$

Proof Construct the following Lyapunov functional for network (33):

$$
\begin{aligned}
V_{4}(t)= & \frac{1}{2} \sum_{\kappa=1}^{m} \frac{c_{\kappa}}{1-\gamma_{\kappa}} \int_{t-\tau_{\kappa}(t)}^{t} \int_{\Theta} e^{T}(\vartheta, h) e(\vartheta, h) d \vartheta d h \\
& +\int_{-\tau}^{0} \int_{t+\rho}^{t} \int_{\Theta} e^{T}(\vartheta, h) e(\vartheta, h) d \vartheta d h d \rho
\end{aligned}
$$




$$
+\frac{1}{2} \sum_{s=1}^{M} \int_{\Theta} e_{s}^{T}(\vartheta, t) e_{s}(\vartheta, t) d \vartheta .
$$

Obviously, $\left(\frac{1}{\sqrt{2}}\|e(\cdot, t)\|\right)^{2} \leqslant V_{4}(t)$ and we can deduce from (36) as follows:

$$
\begin{aligned}
V_{4}(t) \leqslant & \frac{1}{2} \sum_{\kappa=1}^{m} \frac{c_{\kappa}}{1-\gamma_{\kappa}} \int_{t-\tau}^{t} \int_{\Theta} e^{T}(\vartheta, h) e(\vartheta, h) d \vartheta d h \\
& +\tau \int_{t-\tau}^{t} \int_{\Theta} e^{T}(\vartheta, h) e(\vartheta, h) d \vartheta d h \\
& +\frac{1}{2} \sum_{s=1}^{M} \int_{\Theta} e_{s}^{T}(\vartheta, t) e_{s}(\vartheta, t) d \vartheta \\
= & \left(\tau+\frac{1}{2} \sum_{\kappa=1}^{m} \frac{c_{\kappa}}{1-\gamma_{\kappa}}\right) \int_{t-\tau}^{t} \int_{\Theta} e^{T}(\vartheta, h) e(\vartheta, h) d \vartheta d h \\
& +\frac{1}{2} \sum_{s=1}^{M} \int_{\Theta} e_{s}^{T}(\vartheta, t) e_{s}(\vartheta, t) d \vartheta .
\end{aligned}
$$

By calculating the derivative of (36), one has

$$
\begin{aligned}
\dot{V}_{4}(t) \leqslant & \sum_{s=1}^{M} \int_{\Theta} e_{s}^{T}(\vartheta, t)\left(-A e_{s}(\vartheta, t)+\sum_{\kappa=1}^{m} \sum_{j=1}^{M} c_{\kappa} G_{s j}^{\kappa} \Gamma_{\kappa} e_{j}\left(\vartheta, t-\tau_{\kappa}(t)\right)\right. \\
& +B g\left(Y_{s}(\vartheta, t)\right)+B g\left(W_{s}(\vartheta, t)\right)+D \triangle e_{s}(\vartheta, t)-q_{s} e_{s}(\vartheta, t) \\
& \left.-\beta_{s} \frac{\|e(\cdot, t)\|^{2} e_{s}(\vartheta, t)}{\|e(\cdot, t)\|^{2}+\eta(t)}\right) d \vartheta+\tau \int_{\Theta} e^{T}(\vartheta, t) e(\vartheta, t) d \vartheta \\
& -\frac{1}{2} \sum_{\kappa=1}^{m} c_{\kappa} \int_{\Theta} e^{T}\left(\vartheta, t-\tau_{\kappa}(t)\right) e\left(\vartheta, t-\tau_{\kappa}(t)\right) d \vartheta \\
& +\frac{1}{2} \sum_{\kappa=1}^{m} \frac{c_{\kappa}}{1-\gamma_{\kappa}} \int_{\Theta} e^{T}(\vartheta, t) e(\vartheta, t) d \vartheta \\
& -\int_{t-\tau}^{t} \int_{\Theta} e^{T}(\vartheta, h) e(\vartheta, h) d \vartheta d h,
\end{aligned}
$$

where $e\left(\vartheta, t-\tau_{\kappa}(t)\right)=\left(e_{1}^{T}\left(\vartheta, t-\tau_{\kappa}(t)\right), e_{2}^{T}\left(\vartheta, t-\tau_{\kappa}(t)\right), \cdots\right.$, $\left.e_{M}^{T}\left(\vartheta, t-\tau_{\kappa}(t)\right)\right)^{T}$. Obviously,

$$
\begin{aligned}
& \sum_{\kappa=1}^{m} \sum_{s=1}^{M} \sum_{j=1}^{M} c_{\kappa} G_{s j}^{\kappa} e_{s}^{T}(\vartheta, t) \Gamma_{\kappa} e_{j}\left(\vartheta, t-\tau_{\kappa}(t)\right) \\
\leqslant & \frac{1}{2} \sum_{\kappa=1}^{m} c_{\kappa} e^{T}(\vartheta, t)\left(\left(G^{\kappa}\right)^{2} \otimes\left(\Gamma_{\kappa}\right)^{2}\right) e(\vartheta, t) \\
& +\frac{1}{2} \sum_{\kappa=1}^{m} c_{\kappa} e^{T}\left(\vartheta, t-\tau_{\kappa}(t)\right) e\left(\vartheta, t-\tau_{\kappa}(t)\right) .
\end{aligned}
$$

From (38) and (39), we have

$$
\begin{aligned}
\dot{V}_{4}(t) \leqslant & \left.\left.\sum_{s=1}^{M} \int_{\Theta} e_{s}^{T}(\vartheta, t)\left(-A e_{s}(\vartheta, t)+B g\left(Y_{s}(\vartheta, t)\right)+D \triangle e_{s}(\vartheta, t)+\tau\right) I_{n}\right)-(\hat{\beta}+\hat{q}) \otimes I_{n}\right] e(\vartheta, t) d \vartheta+\sigma(\tau \\
& \left.\left.+B g\left(W_{s}(\vartheta, t)\right)-q_{s} e_{s}(\vartheta, t)-\beta_{s} \frac{\|e(\cdot, t)\|^{2} e_{s}(\vartheta, t)}{\|e(\cdot, t)\|^{2}+\eta(t)}\right) d \vartheta \quad+\frac{1}{2} \sum_{\kappa=1}^{m} \frac{c_{\kappa}}{1-\gamma_{\kappa}}\right) \int_{t-\tau}^{t} \int_{\Theta} e^{T}(\vartheta, h) e(\vartheta, h) d \vartheta d h
\end{aligned}
$$

Combine (37) with (40), one has

$$
\begin{aligned}
& \dot{V}_{4}(t)+\sigma V_{4}(t) \\
\leqslant & \int_{\Theta} e^{T}(\vartheta, t)\left[\frac{1}{2} \sum_{\kappa=1}^{m} c_{\kappa}\left(G^{\kappa}\right)^{2} \otimes\left(\Gamma_{\kappa}\right)^{2}+I_{M} \otimes(-A\right. \\
& +\frac{1}{2}\left(B B^{T}+F\right)-\sum_{r=1}^{p} \frac{1}{\iota_{r}^{2}} D+\left(\frac{1}{2} \sum_{\kappa=1}^{m} \frac{c_{\kappa}}{1-\gamma_{\kappa}}\right.
\end{aligned}
$$




$$
\begin{aligned}
& +\frac{\sigma}{2} \sum_{s=1}^{M} \int_{\Theta} e_{s}^{T}(\vartheta, t) e_{s}(\vartheta, t) d \vartheta+\beta \eta(t) \\
& -\int_{t-\tau}^{t} \int_{\Theta} e^{T}(\vartheta, h) e(\vartheta, h) d \vartheta d h \\
& \leqslant\left(\tau \sigma-1+\frac{\sigma}{2} \sum_{\kappa=1}^{m} \frac{c_{\kappa}}{1-\gamma_{\kappa}}\right) \int_{t-\tau}^{t} \int_{\Theta} e^{T}(\vartheta, h) e(\vartheta, h) d \vartheta d h \\
& +\int_{\Theta} e^{T}(\vartheta, t)\left[\frac{1}{2} \sum_{\kappa=1}^{m} c_{\kappa}\left(G^{\kappa}\right)^{2} \otimes\left(\Gamma_{\kappa}\right)^{2}+I_{M} \otimes(-A\right. \\
& +\frac{1}{2}\left(B B^{T}+F\right)+\left(\frac{\sigma}{2}+\tau+\frac{1}{2} \sum_{\kappa=1}^{m} \frac{c_{\kappa}}{1-\gamma_{\kappa}}\right) I_{n} \\
& \left.\left.-\sum_{r=1}^{p} \frac{1}{\iota_{r}^{2}} D\right)-(\hat{\beta}+\hat{q}) \otimes I_{n}\right] e(\vartheta, t) d \vartheta+\beta \eta(t) .
\end{aligned}
$$

According to (34) and (35), we can obtain

$$
\dot{V}_{4}(t)+\sigma V_{4}(t) \leqslant \beta \eta(t) .
$$

Take $\alpha_{1}=\frac{1}{\sqrt{2}}, \alpha_{2}=\beta$, it is easy to obtain that network (33) is $\psi$-type stable with convergence rate $\frac{\sigma}{2}$. Then, the drive system (31) and response system (32) reach general decay anti-synchronization.

Remark 4 As is well known, linear matrix inequality, matrix measure theory, stability theory method are commonly used methods for studying the synchronization of NNs [5, 13, 23, 24,40]. In [5], Cao and Wan presented several stability and synchronization criteria for inertial BAM NNs by using matrix measure strategies. However, the majority of network models in above-mentioned results are described by ordinary differential equations. Actually, reaction-diffusion effects are very important and unavoidable in NNs once the electrons transport in inhomogeneous magnetic field. Therefore, it is necessary and meaningful to study the CNNs with reactiondiffusion terms $[31,33,39,43]$, which are described by partial differential equations, i.e., the state of each neuron is not only dependent on the time variable, but also intensively dependent on space variable. Unfortunately, there are no results reported on the decay anti-synchronization of CRDNNs with multi-weights. In this section, the definition of $\psi$-type stability for MWCRDNNs is firstly given, which is equivalent to decay anti-synchronization of the considered drive-response systems of MWCRDNNs. Then, several decay anti-synchronization criteria for MWCRDNNs are established by designing appropriate controller. Actually, the main difficulty for obtaining decay anti-synchronization conditions in our paper comes from the reaction-diffusion terms and multi-weighted terms, which cannot be dealt with by those traditional techniques used in the above literatures. Due to this, by constructing new Lyapunov functionals, and employing Green's formula, Lemmas 1 and 2 , and some other inequality techniques, we establish some conditions for achieving decay anti-synchronization of MWCRDNNs in Theorems 3 and 4, which are dependent on the reaction-diffusion terms and multiweighted terms.

\section{Numerical Examples}

Example 5.1. Consider the following MWCNN with delayed coupling:

$$
\begin{aligned}
\dot{Y}_{s}(t)= & -A Y_{s}(t)+B g\left(Y_{s}(t)\right)+0.2 \sum_{j=1}^{6} G_{s j}^{1} \Gamma_{1} Y_{j}\left(t-\tau_{1}(t)\right) \\
& +0.2 \sum_{j=1}^{6} G_{s j}^{2} \Gamma_{2} Y_{j}\left(t-\tau_{2}(t)\right)+0.1 \sum_{j=1}^{6} G_{s j}^{3} \Gamma_{3} Y_{j}(t \\
& \left.-\tau_{3}(t)\right), \quad s=1,2, \cdots, 6
\end{aligned}
$$

where $g_{i}(\delta)=\frac{|\delta+1|-|\delta-1|}{8}(i=1,2,3), A=\operatorname{diag}(0.3,0.4,0.6)$, $\Gamma_{1}=\operatorname{diag}(0.2,0.3,0.1), \Gamma_{2}=\operatorname{diag}(0.1,0.2,0.1), \Gamma_{3}=$ $\operatorname{diag}(0.2,0.2,0.3), \tau_{1}(t)=\frac{1}{20}-\frac{1}{20} e^{-t}, \tau_{2}(t)=\frac{1}{20}-$ $\frac{1}{30} e^{-t}, \tau_{3}(t)=\frac{1}{20}-\frac{1}{40} e^{-t}, \tau=\frac{1}{20}, \gamma_{1}=\frac{1}{20}, \gamma_{2}=$ $\frac{1}{30}, \gamma_{3}=\frac{1}{40}$, choose the following matrices as $B, G^{1}, G^{2}$ and $G^{3}$ respectively

$$
\begin{gathered}
B=\left(\begin{array}{ccccc}
0.3 & 0 & 0.1 \\
0.1 & 0.2 & 0 \\
0.1 & 0 & 0.4
\end{array}\right), \\
G^{1}=\left(\begin{array}{cccccc}
-0.6 & 0.2 & 0.1 & 0.1 & 0 & 0.2 \\
0.2 & -0.7 & 0.3 & 0 & 0.2 & 0 \\
0.1 & 0.3 & -0.6 & 0 & 0.1 & 0.1 \\
0.1 & 0 & 0 & -0.5 & 0.2 & 0.2 \\
0 & 0.2 & 0.1 & 0.2 & -0.5 & 0 \\
0.2 & 0 & 0.1 & 0.2 & 0 & -0.5
\end{array}\right), \\
G^{2}=\left(\begin{array}{cccccc}
-0.5 & 0 & 0.3 & 0.1 & 0.1 & 0 \\
0 & -0.4 & 0.2 & 0.1 & 0 & 0.1 \\
0.3 & 0.2 & -0.8 & 0.1 & 0 & 0.2 \\
0.1 & 0.1 & 0.1 & -0.4 & 0 & 0.1 \\
0.1 & 0 & 0 & 0 & -0.3 & 0.2 \\
0 & 0.1 & 0.2 & 0.1 & 0.2 & -0.6
\end{array}\right), \\
G^{3}=\left(\begin{array}{cccccc}
-0.6 & 0.1 & 0 & 0.2 & 0.2 & 0.1 \\
0.1 & -0.5 & 0.2 & 0.1 & 0.1 & 0 \\
0 & 0.2 & -0.4 & 0 & 0 & 0.2 \\
0.2 & 0.1 & 0 & -0.6 & 0.3 & 0 \\
0.2 & 0.1 & 0 & 0.3 & -0.8 & 0.2 \\
0.1 & 0 & 0.2 & 0 & 0.2 & -0.5
\end{array}\right) .
\end{gathered}
$$

Obviously, $g_{i}(\cdot)(i=1,2,3)$ satisfies Assumption 1 with $F_{i}=0.25$. Consider (41) as the drive system, then its 
corresponding response system is shown as follows:

$$
\begin{aligned}
\dot{W}_{s}(t)= & -A W_{s}(t)+B g\left(W_{s}(t)\right)+0.2 \sum_{j=1}^{6} G_{s j}^{1} \Gamma_{1} W_{j}\left(t-\tau_{1}(t)\right) \\
& +0.2 \sum_{j=1}^{6} G_{s j}^{2} \Gamma_{2} W_{j}\left(t-\tau_{2}(t)\right)+0.1 \sum_{j=1}^{6} G_{s j}^{3} \Gamma_{3} W_{j}\left(t_{\{v}^{A}\right. \\
& \left.-\tau_{3}(t)\right)+u_{s}(t), s=1,2, \cdots, 6,
\end{aligned}
$$

the parameters in the controller (4) are chosen as follows: $\hat{q}=\operatorname{diag}(0.8,0.6,0.4,0.2,0.5,0.2), \hat{\beta}=\operatorname{diag}(0.3,0.5$, $0.4,0.3,0.2,0.5)$ and $\eta(t)=e^{-0.2 t}$. Then the nonlinear controller (4) is transferred as follows:

$$
\left\{\begin{array}{l}
u_{1}(t)=-0.8 e_{1}(t)-0.3 \frac{\|e(t)\|^{2} e_{1}(t)}{\|e(t)\|^{2}+e^{-0.2 t}}, \\
u_{2}(t)=-0.6 e_{2}(t)-0.5 \frac{\|e(t)\|^{2} e_{2}(t)}{\|e(t)\|^{2}+e^{-0.2 t}}, \\
u_{3}(t)=-0.4 e_{3}(t)-0.4 \frac{\|e(t)\|^{2} e_{3}(t)}{\|e(t)\|^{2}+e^{-0.2 t}}, \\
u_{4}(t)=-0.2 e_{4}(t)-0.3 \frac{\|e(t)\|^{2} e_{4}(t)}{\|e(t)\|^{2}+e^{-0.2 t}}, \\
u_{5}(t)=-0.5 e_{5}(t)-0.2 \frac{\|e(t)\|^{2} e_{5}(t)}{\|e(t)\|^{2}+e^{-0.2 t}}, \\
u_{6}(t)=-0.2 e_{6}(t)-0.5 \frac{\|e(t)\|^{2} e_{6}(t)}{\|e(t)\|^{2}+e^{-0.2 t}} .
\end{array}\right.
$$

The other parameters in (42) are defined the same as those in (41). We choose $\sigma=0.02$, in other words, the convergence rate is $\frac{\sigma}{2}=0.01$. Through a simple operation based on the above parameters, we have

$$
\begin{aligned}
\lambda\left(\Phi_{2}\right)= & \{-1.1714,-1.1726,-0.2960,-1.0269,-1.0229, \\
& -0.4269,-0.8963,-0.8943,-0.8722,-0.4963, \\
& -0.4952,-0.7699,-0.7729,-0.7237,-0.5718, \\
& -0.5953,-0.6272,-0.6256\}, \\
\Lambda_{2}= & 0.0062<1,
\end{aligned}
$$

which satisfy the conditions (14) and (15).

According to Theorem 2, the systems (41) and (42) reach general decay anti-synchronization under the nonlinear controller (43). The simulation result is shown in Fig. 1.

Example 5.2. Consider the following MWCRDNN with delayed coupling:

$$
\begin{aligned}
\frac{\partial Y_{s}(\vartheta, t)}{\partial t}= & -A Y_{s}(\vartheta, t)+D \triangle Y_{s}(\vartheta, t)+B g\left(Y_{s}(\vartheta, t)\right) \\
& +0.1 \sum_{j=1}^{6} G_{s j}^{1} \Gamma_{1} Y_{j}\left(\vartheta, t-\tau_{1}(t)\right) \\
& +0.2 \sum_{j=1}^{6} G_{s j}^{2} \Gamma_{2} Y_{j}\left(\vartheta, t-\tau_{2}(t)\right)
\end{aligned}
$$

$$
+0.3 \sum_{j=1}^{6} G_{s j}^{3} \Gamma_{3} Y_{j}\left(\vartheta, t-\tau_{3}(t)\right)
$$

where $s=1,2, \cdots, 6, g_{i}(\delta)=\frac{|\delta+1|-|\delta-1|}{4}(i=1,2,3)$, $A=\operatorname{diag}(0.4,0.6,0.5), D=\operatorname{diag}(0.3,0.2,0.4), \Theta=$ $\{\vartheta \mid-1<\vartheta<1\}, \Gamma_{1}=\operatorname{diag}(0.1,0.3,0.2), \Gamma_{2}=\operatorname{diag}(0.4$, $0.2,0.2), \Gamma_{3}=\operatorname{diag}(0.1,0.1,0.2), \tau_{1}(t)=\frac{1}{20}-\frac{1}{10} e^{-t}, \tau_{2}($ $t)=\frac{1}{20}-\frac{1}{20} e^{-t}, \tau_{3}(t)=\frac{1}{20}-\frac{1}{30} e^{-t}, \tau=\frac{1}{20}, \gamma_{1}=$ $\frac{1}{10}, \gamma_{2}=\frac{1}{20}, \gamma_{3}=\frac{1}{30}$, choose the following matrices as $B, G^{1}, G^{2}$ and $G^{3}$ respectively

$$
\begin{gathered}
B=\left(\begin{array}{ccccc}
0.4 & 0.1 & 0.2 \\
0 & 0.2 & 0 \\
0.1 & 0 & 0.3
\end{array}\right), \\
G^{1}=\left(\begin{array}{cccccc}
-0.6 & 0.1 & 0.2 & 0.2 & 0.1 & 0 \\
0.1 & -0.7 & 0 & 0.3 & 0.1 & 0.2 \\
0.2 & 0 & -0.3 & 0 & 0 & 0.1 \\
0.2 & 0.3 & 0 & -0.7 & 0.2 & 0 \\
0.1 & 0.1 & 0 & 0.2 & -0.5 & 0.1 \\
0 & 0.2 & 0.1 & 0 & 0.1 & -0.4
\end{array}\right), \\
G^{2}=\left(\begin{array}{cccccc}
-0.4 & 0.1 & 0 & 0.2 & 0.1 & 0 \\
0.1 & -0.5 & 0.3 & 0.1 & 0 & 0 \\
0 & 0.3 & -0.6 & 0.1 & 0 & 0.2 \\
0.2 & 0.1 & 0.1 & -0.5 & 0 & 0.1 \\
0.1 & 0 & 0 & 0 & -0.2 & 0.1 \\
0 & 0 & 0.2 & 0.1 & 0.1 & -0.4
\end{array}\right), \\
G^{3}=\left(\begin{array}{cccccc}
-0.6 & 0.3 & 0.1 & 0 & 0.2 & 0 \\
0.3 & -0.8 & 0.2 & 0.1 & 0.1 & 0.1 \\
0.1 & 0.2 & -0.6 & 0.1 & 0 & 0.2 \\
0 & 0.1 & 0.1 & -0.4 & 0.2 & 0 \\
0.2 & 0.1 & 0 & 0.2 & -0.7 & 0.2 \\
0 & 0.1 & 0.2 & 0 & 0.2 & -0.5
\end{array}\right) .
\end{gathered}
$$

Obviously, $g_{i}(\cdot)(i=1,2,3)$ satisfies Assumption 1 with $F_{i}=0.5$. Consider (44) as the drive system, then its corresponding response system is shown as follows:

$$
\begin{aligned}
\frac{\partial W_{s}(\vartheta, t)}{\partial t}= & -A W_{s}(\vartheta, t)+D \triangle W_{s}(\vartheta, t)+B g\left(W_{s}(\vartheta, t)\right) \\
& +0.1 \sum_{j=1}^{6} G_{s j}^{1} \Gamma_{1} W_{j}\left(\vartheta, t-\tau_{1}(t)\right)+u_{s}(\vartheta, t) \\
& +0.2 \sum_{j=1}^{6} G_{s j}^{2} \Gamma_{2} W_{j}\left(\vartheta, t-\tau_{2}(t)\right) \\
& +0.3 \sum_{j=1}^{6} G_{s j}^{3} \Gamma_{3} W_{j}\left(\vartheta, t-\tau_{3}(t)\right)
\end{aligned}
$$

where $s=1,2, \cdots, 6$, the parameters in the controller (24) are chosen as follows: $\hat{q}=\operatorname{diag}(0.7,0.6,0.5,0.6,0.4,0.2)$, $\hat{\beta}=\operatorname{diag}(0.2,0.3,0.6,0.4,0.5,0.3)$ and $\eta(t)=e^{-0.1 t}$. Then the nonlinear controller (24) is transferred as fol- 

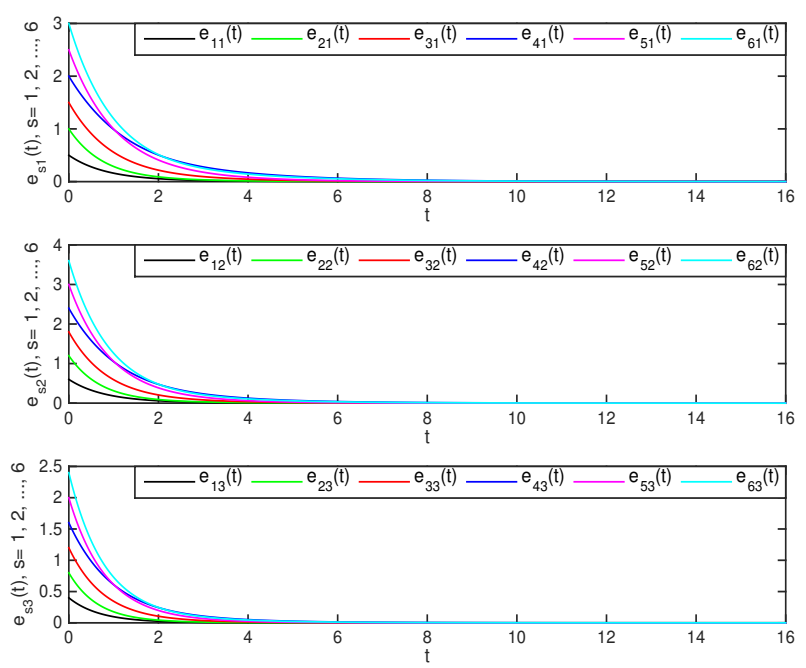

Fig. $1 e_{s j}(t), s=1,2, \cdots, 6, j=1,2,3$.

lows:

$$
\left\{\begin{array}{l}
u_{1}(\vartheta, t)=-0.7 e_{1}(\vartheta, t)-0.2 \frac{\|e(\cdot, t)\|^{2} e_{1}(\vartheta, t)}{\|e(\cdot, t)\|^{2}+e^{-0.1 t}}, \\
u_{2}(\vartheta, t)=-0.6 e_{2}(\vartheta, t)-0.3 \frac{\|e(\cdot, t)\|^{2} e_{2}(\vartheta, t)}{\|e(\cdot, t)\|^{2}+e^{-0.1 t}}, \\
u_{3}(\vartheta, t)=-0.5 e_{3}(\vartheta, t)-0.6 \frac{\|e(\cdot, t)\|^{2} e_{3}(\vartheta, t)}{\|e(\cdot, t)\|^{2}+e^{-0.1 t}}, \\
u_{4}(\vartheta, t)=-0.6 e_{4}(\vartheta, t)-0.4 \frac{\|e(\cdot, t)\|^{2} e_{4}(\vartheta, t)}{\|e(\cdot, t)\|^{2}+e^{-0.1 t}}, \\
u_{5}(\vartheta, t)=-0.4 e_{5}(\vartheta, t)-0.5 \frac{\|e(\cdot, t)\|^{2} e_{5}(\vartheta, t)}{\|e(\cdot, t)\|^{2}+e^{-0.1 t}}, \\
u_{6}(\vartheta, t)=-0.2 e_{6}(\vartheta, t)-0.3 \frac{\|e(\cdot, t)\|^{2} e_{6}(\vartheta, t)}{\|e(\cdot, t)\|^{2}+e^{-0.1 t}} .
\end{array}\right.
$$

The other parameters in (45) are defined the same as those in (44). We choose $\sigma=0.2$, in other words, the convergence rate is $\frac{\sigma}{2}=0.1$. Through a simple operation based on the above parameters, we have

$$
\begin{aligned}
\lambda\left(\Phi_{4}\right)= & \{-0.3649,-0.5621,-0.6401,-0.7607,-0.7647, \\
& -0.7676,-1.2384,-0.8634,-1.1396,-1.1611, \\
& -1.0600,-1.0414,-1.0343,-1.0380,-0.9584, \\
& -0.9624,-0.9607,-0.9606\}, \\
\Lambda_{4}= & 0.0732<1,
\end{aligned}
$$

which satisfy the conditions (34) and (35).

According to Theorem 4, the systems (44) and (45) reach general decay anti-synchronization under the nonlinear controller (46). The simulation result is displayed in Fig. 2.
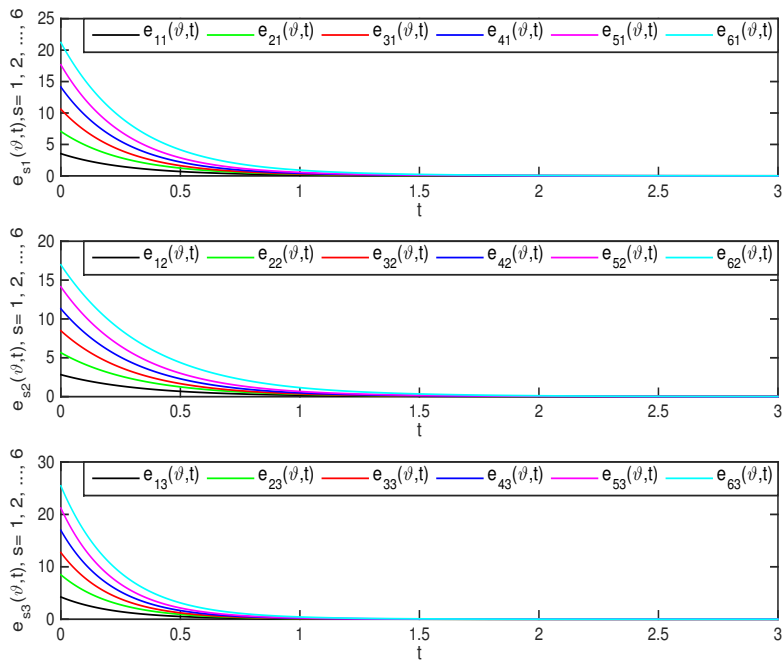

Fig. $2 e_{s j}(\vartheta, t), s=1,2, \cdots, 6, j=1,2,3$.

\section{Conclusion}

This paper has proposed the concept of general decay anti-synchronization by introducing $\psi$-type stability and $\psi$-type function. Then, the decay anti-synchronization for MWCNNs and MWCRDNNs has been investigated, respectively. By utilizing Lyapunov functional approach, some inequality techniques, as well as the construction of nonlinear controllers, several novel criteria for ensuring decay anti-synchronization for these two networks have been obtained. Additionally, we also have considered the decay anti-synchronization for these networks with delayed coupling. Finally, two examples have been given to verify the correctness and validity for our results.

\section{Acknowledgement}

The authors would like to thank the Associate Editor and anonymous reviewers for their valuable comments and suggestions. They also wish to express their sincere appreciation to Prof. Jinliang Wang for the fruitful discussions and valuable suggestions which helped to improve this paper. This work was supported in part by the Natural Science Foundation of Tianjin City under Grant 18JCQNJC74300, in part by the National Natural Science Foundation of China under Grant 61773285, and in part by Chinese Scholarship Council. Dr E. Yang is supported in part under the RSE-NNSFC Joint Project (2017-2019) under Grant 6161101383 with China University of Petroleum (East China). 
Conflict of Interest The authors declare that they have no conflict of interest.

\section{References}

1. Abdurahman A (2018) New results on the general decay synchronization of delayed neural networks with general activation functions. Neurocomputing 275: 2505-2511

2. An XL, Zhang L, Li YZ, Zhang JG (2014) Synchronization analysis of complex networks with multi-weights and its application in public traffic network. Physica A 412: 149156

3. An XL, Zhang L, Zhang JG (2015) Research on urban public traffic network with multi-weights based on single bus transfer junction. Physica A 436: 748-755

4. Asadia E, Silva MG, Antunes CH, Diasc L, Glicksman L (2014) Multi-objective optimization for building retrofit: a model using genetic algorithm and artificial neural network and an application. Energ Buildings 81: 444-456

5. Cao JD, Wan Y (2014) Matrix measure strategies for stability and synchronization of inertial BAM neural network with time delays. Neural Netw 53: 165-172

6. Gao F, Huang T, Sun JP, Wang J, Hussain A, Yang E (2018) A new algorithm of SAR image target recognition based on improved deep convolutional neural network. Cogn Comput. https://doi.rog/10.1007/s12559-018-9563-$$
\text { z }
$$

7. Geng X, Zhou ZH, Smith-Miles K (2008) Individual stable space: an approach to face recognition under uncontrolled conditions. IEEE Trans Neural Netw 19: 1354-1368

8. Hou J, Huang YL, Ren SY (2019) Anti-synchronization analysis and pinning control of multi-weighted coupled neural networks with and without reaction-diffusion terms. Neurocomputing 330: 78-93

9. Huang YL, Qiu SH, Ren SY (2019) Finitetime synchronization and passivity of coupled memristive neural networks. Int $\mathrm{J}$ Control. http://doi.rog/10.1080/00207179.2019.1566640

10. Huang YL, Qiu SH, Ren SY, Zheng ZW (2018) Fixedtime synchronization of coupled Cohen-Grossberg neural networks with and without parameter uncertainties. Neurocomputing 315: 157-168

11. Huang YL, Wang SX, Ren SY (2017) Pinning exponential synchronization and passivity of coupled delayed reaction-diffusion neural networks with and without parametric uncertainties. Int $\mathrm{J}$ Control. https://doi.org/10.1080/00207179.2017.1384575

12. Hu C, Jiang HJ, Teng ZD (2010) Impulsive control and synchronization for delayed neural networks with reactiondiffusion terms. IEEE Trans Neural Netw 21: 67-81

13. Hu JQ, Cao JD, Alofi A, AL-Mazrooei A, Elaiw A (2015) Pinning synchronization of coupled inertial delayed neural networks. Cognit Neurodyn 9: 341-350

14. Hien LV, Phat VN, Trinh H (2015) New generalized Halanay inequalities with applications to stability of nonlinear non-autonomous time-delay systems. Nonlinear Dyn 82:563-575

15. Lin SH, Kung SY, Lin LJ (1997) Face recognition / detection by probabilistic decision-based neural network. IEEE Trans Neural Netw 8: 114-132

16. Liu D, Zhu S, Sun K (2018) Global antisynchronization of complex-valued memristive neural networks with time delays. IEEE Trans Cyber. https://doi.org/10.1109/TCYB.2018.2812708
17. Liu YJ, Park JH, Guo BZ, Fang F, Zhou FN (2018) Event-triggered dissipative synchronization for Markovian jump neural networks with general transition probabilities. Int J Robust Nonlin 28: 3893-3908

18. Lu JG (2008) Global exponential stability and periodicity of reaction-diffusion delayed recurrent neural networks with dirichlet boundary conditions. Chaos Solitons Fractals 35: 116-125

19. Ma CQ, Zhang JF (2012) On formability of linear continuous multi-agent systems. J Syst Sci Complex 25: 13-29

20. Ma CQ, Zhao WW, Zhao YB (2018) Bipartite consensus of discrete-time double-integrator multi-agent systems with measurement noise. J Syst Sci Complex 31: 1525-1540

21. Mahmoud EE (2012) Adaptive anti-lag synchronization of two identical or non-identical hyperchaotic complex nonlinear systems with uncertain parameters. J Frankl Inst 349: 1247-1266

22. Ma Q, Feng G, Xu SY (2013) Delay-dependent stability criteria for reaction-diffusion neural networks with timevarying delays. IEEE Trans Cyber 43: 1913-1920

23. Qi DL, Liu MQ, Qiu MK, Zhang SL (2010) Exponential $\mathcal{H}_{\infty}$ synchronization of general discrete-time chaotic neural networks with or without time delays. IEEE Trans Neural Netw 21: 1358-1365

24. Ren FL, Cao JD (2009) Anti-synchronization of stochastic perturbed delayed chaotic neural networks. Neural Comput Appl 18: 515-521

25. Sader M, Abdurahman A, Jiang HJ (2018) General decay synchronization of delayed BAM neural networks via nonlinear feedback control. Appl Math Comput 337: 302314

26. Sakthivel R, Vadivel P, Mathiyalaganc K, Arunkumar A, Sivachitra M (2015) Design of state estimator for bidirectional associative memory neural networks with leakage delays. Inf Sci 296: 263-274

27. Shen H, Huo SC, Cao JD, Huang TW (2019) Generalized state estimation for Markovian coupled networks under round-robin protocol and redundant channels. IEEE Trans Cyber 49: 1292-1301

28. Tang Z, Park JH, Feng JW (2018) Impulsive effects on quasi-synchronization of neural networks with parameter mismatches and time-varying delay. IEEE Trans Neural Netw Learn Syst 29: 908-919

29. Venetianer PL, Roska T (1998) Image compression by cellular neural networks. IEEE Trans Circuits Syst I Fundam Theory Appl 45: 205-215

30. Wang H, Duan S, Huang T, Wang L, Li C (2017) Exponential stability of complex-valued memristive recurrent neural networks. IEEE Trans Neural Netw Learn Sys 28: 766-771

31. Wang JL, Wu HN (2014) Synchronization and adaptive control of an array of linearly coupled reaction-diffusion neural networks with hybrid coupling. IEEE Trans Cyber 44: $1350-1361$

32. Wang JL, Wu HN, Guo L (2011) Passivity and stability analysis of reaction-diffusion neural networks with dirichlet boundary conditions. IEEE Trans Neural Netw 22: 2105-2116

33. Wang JL, Wu HN, Huang TW, Ren SY (2015) Passivity and synchronization of linearly coupled reaction-diffusion neural networks with adaptive coupling. IEEE Trans Cyber 45: 1942-1952

34. Wang JL, Xu M, Wu HN, Huang TW (2018) Finitetime passivity of coupled neural networks with multiple weights. IEEE Trans Netw Sci Eng. 5: 184-197

35. Wang LM, Shen Y, Zhang GD (2016) Synchronization of a class of switched neural networks with time-varying 
delays via nonlinear feedback control. IEEE Trans Cyber 46: $2300-2310$

36. Wang LM, Shen Y, Zhang GD (2016) General decay synchronization stability for a class of delayed chaotic neural networks with discontinuous activations. Neurocomputing 179: $169-175$

37. Wang LM, Shen Y, Zhang GD (2016) Synchronization of a class of switched neural networks with time-varying delays via nonlinear feedback control. IEEE Trans Cyber 46: $2300-2310$

38. Wang LM, Zeng ZG, Ge MF, Hu JH (2018) Global stabilization analysis of inertial memristive recurrent neural networks with discrete and distributed delays. Neural Netw 105: 65-74

39. Wang Q, Wang JL, Ren SY, Huang YL (2018) Analysis and adaptive control for lag $\mathcal{H}_{\infty}$ synchronization of coupled reaction-diffusion neural networks. Neurocomputing 319: $144-154$

40. Wang WP, Li LX, Peng HP, Wang WN, Kurths J, Xiao JH, Yang YX (2016) Anti-synchronization of coupled memristive neutral-type neural networks with mixed time-varying delays via randomly occurring control. Nonlinear Dyn 4: 2143-2155

41. Wu FG, Hu SG (2012) Razumikhin-type theorems on general decay stability and robustness for stochastic functional differential equations. Int J Robust Nonlin 22: 763777

42. Wu W, Chen TP (2008) Global synchronization criteria of linearly coupled neural network systems with timevarying coupling. IEEE Trans Neural Netw 19: 319-332

43. Wu YZ, Liu L, Hu JP, Feng G (2018) Adaptive antisynchronization of multilayer reaction-diffusion neural networks. IEEE Trans Neural Netw Learn Syst 29: 807-818

44. Yang CB, Huang TZ (2014) Improved stability criteria for a class of neural networks with variable delays and impulsive perturbations. Appl Math Comput 243: 923-935

45. Yue ZY, Gao F, Xiong QX, Wang J, Huang T, Yang E, Zhou HY (2019) A novel semi-supervised convolutional neural network method for synthetic aperture radar image recognition. Cogn Comput. http://doi.rog/10.1007/s12559-019-09639-x

46. Zhang CK, He Y, Jiang L, Wang QG, Wu M (2017) Stability analysis of discrete-time neural networks with timevarying delay via an extended reciprocally convex matrix inequality. IEEE Trans Cyber 47: 3040-3049

47. Zhang FH, Zeng ZG (2018) Multiple $\psi$-type stability of Cohen-Grossberg neural networks with unbounded time-varying delays. IEEE Trans Syst Man Cyber. https://doi.org/10.1109/TSMC.2018.2876003

48. Zhang RM, Zeng DQ, Park JH, Zhong SM, Yu YB (2018) Novel discontinuous control for exponential synchronization of memristive recurrent neural networks with heterogeneous time-varying delays. J Frankl Inst 355: 2826-2848

49. Zhang W, Li CD, Huang TW, He X (2015) Synchronization of memristor-based coupling recurrent neural networks with time-varying delays and impulses. IEEE Trans Neural Netw Learn Syst 26: 3308-3313

50. Zhang ZQ, Ren L (2018) New sufficient conditions on global asymptotic synchronization of inertial delayed neural networks by using integrating inequality techniques. Nonlinear Dyn. https://doi.org/10.1007/s11071-018-46035

51. Zhang ZQ, Cao JD (2018) Novel finite-time synchronization criteria for inertial neural networks with time delays via integral inequality method. IEEE Trans Neural Netw Learn Syst. https://doi.org/10.1109/TNNLS.2018.2868800
52. Zhang ZQ, Li AL, Yu SH (2018) Finite-time synchronization for delayed complex-valued neural networks via integrating inequality method. Neurocomputing 318: 248260

53. Zhao HY, Zhang Q (2011) Global impulsive exponential anti-synchronization of delayed chaotic neural networks. Neurocomputing 74: 563-567

54. Zhao YP, He P, Nik HS, Ren J (2015) Robust adaptive synchronization of uncertain complex networks with multiple time-varying coupled delays. Complexity 20: 62-73

55. Zheng MW, Li LX, Peng HP, Xiao JH, Yang YX, Zhang YP, Zhao H (2019) General decay synchronization of complex multi-links time-varying dynamic network. Commun Nonlinear Sci Numer Simul 67: 108-123

56. Zheng ZW, Huang YT, Xie LH, Zhu B (2018) Adaptive trajectory tracking control of a fully actuated surface vessel with asymmetrically constrained input and output. IEEE Trans Contr Syst T 26: 1851-1859

57. Zheng ZW, Sun L, Xie LH (2018) Error constrained LOS path following of a surface vessel with actuator saturation and faults. IEEE Trans Syst Man Cybern Syst 48: 21682216 